\title{
SPATIAL AND TEMPORAL VARIATIONS OF K Ca II LINE PROFILE SHAPES IN DIFFERENT STRUCTURES OF THE SOLAR CHROMOSPHERE. II. DETERMINATION TECHNIQUE AND CORRELATION RELATIONSHIPS BETWEEN THE K Ca II LINE PARAMETERS FOR $K_{1}$ AND $K_{2}$ FEATURES
}

\author{
I.P. Turova \\ Institute of Solar-Terrestrial Physics SB RAS, \\ Irkutsk,Russia,turova@iszf.irk.ru
}

\section{S.A. Grigoryeva}

Institute of Solar-Terrestrial Physics SB RAS,

Irkutsk, Russia,sgrig@iszf.irk.ru

\author{
O.A. Ozhogina \\ Institute of Solar-Terrestrial Physics SB RAS \\ Irkutsk,Russia,ozhog@iszf.irk.ru
}

\begin{abstract}
We have studied two regions located at the base of a coronal hole. For the $\mathrm{K}_{1}$ intensity minima and $\mathrm{K}_{2}$ peaks, which form between the upper photosphere and the lower chromosphere and in the lower chromosphere respectively, a number of Ca II line parameters have been computed. We have improved the determination technique for $\Delta \lambda_{\mathrm{K} 1 \mathrm{v}}, \Delta \lambda_{\mathrm{K} 1 \mathrm{r}}, \Delta \lambda_{\mathrm{K} 2 \mathrm{v}}, \Delta \lambda_{\mathrm{K} 2 \mathrm{r}}$ line profile shifts, including certain cases when their direct determination was complicated. We have determined $I_{\mathrm{K} 1 \mathrm{v}}, I_{\mathrm{K} 1 \mathrm{r}}, I_{\mathrm{K} 2 \mathrm{v}}, I_{\mathrm{K} 2 \mathrm{r}}$ intensities, $\mathrm{K}_{1}$ minima and $\mathrm{K}_{2}$ peak separations $S E P_{\mathrm{K} 1}=\Delta \lambda_{\mathrm{K} 1 \mathrm{r}}-\Delta \lambda_{\mathrm{K} 1 \mathrm{v}}, \quad S E P_{\mathrm{K} 2}=\Delta \lambda_{\mathrm{K} 2 \mathrm{r}}-$ $\Delta \lambda_{\mathrm{K} 2 \mathrm{v}}$ respectively. We have constructed scatter plots and have computed correlation relationships between parameters relating to different levels of the atmosphere.

We have obtained the following results.

The intensities observed in the lower and middle chromosphere are connected closer than intensities related to the upper photosphere and middle chromosphere.
\end{abstract}

The structures with a stronger magnetic field are brighter at the upper photosphere and lower chromo- sphere levels as compared to the structures with a weaker magnetic field.

$\mathrm{K}_{1}$ minima separations are of greater value for the structures with a stronger magnetic field relative to the structures with a weaker magnetic field, whereas $\mathrm{K}_{2}$ peak separations demonstrate the opposite behavior. They are lower for the structures with a stronger magnetic field. It is true not only for the chosen structures belonging to quiet regions but also for the plage, though we need additional statistics for plages.

The relation between shifts of $\mathrm{K}_{1}$ minima and $\mathrm{K}_{2}$ peak intensities for violet and red wings appeared to be weak. This may be due to the considerable contribution of random movements to the velocity field at the upper photosphere and lower chromosphere levels or due to different forming levels for the profile violet and red wings.

Keywords: photosphere, chromosphere, K Ca II line profiles.

\section{OBSERVATIONS AND DATA REDUCTION}

Observations used in this paper have been described in Paper I and in [Grigoryeva et al., 2016]. Chromospheric structures were identified from the central intensity of the K Ca II line. Figure 1, $a, b$, taken from Paper I, shows the variation of the time averaged central intensity of the $\mathrm{K}$ line along the spectrograph slit for S25W17 and S25W12. The regions are located at the base of a coronal hole. The chromospheric structures investigated in Paper I (see also [Teplitskaya et al., 2006; Grigoryeva et al., 2016]) are denoted by letters and numbers. The selected chromospheric structures are designated as follows:

«n» - bright network structures («network»);

«ne» - an enhanced network;

«c» - dark internetwork structures («internetWork»);

«b» - a plage;

«p» - a structure with low brightness, which is observed in frames of one of the two series;

$\langle\mathrm{f}\rangle$ - areas on boundaries of the selected structures; also to involve a number of other correlations not cluded in Paper I. 

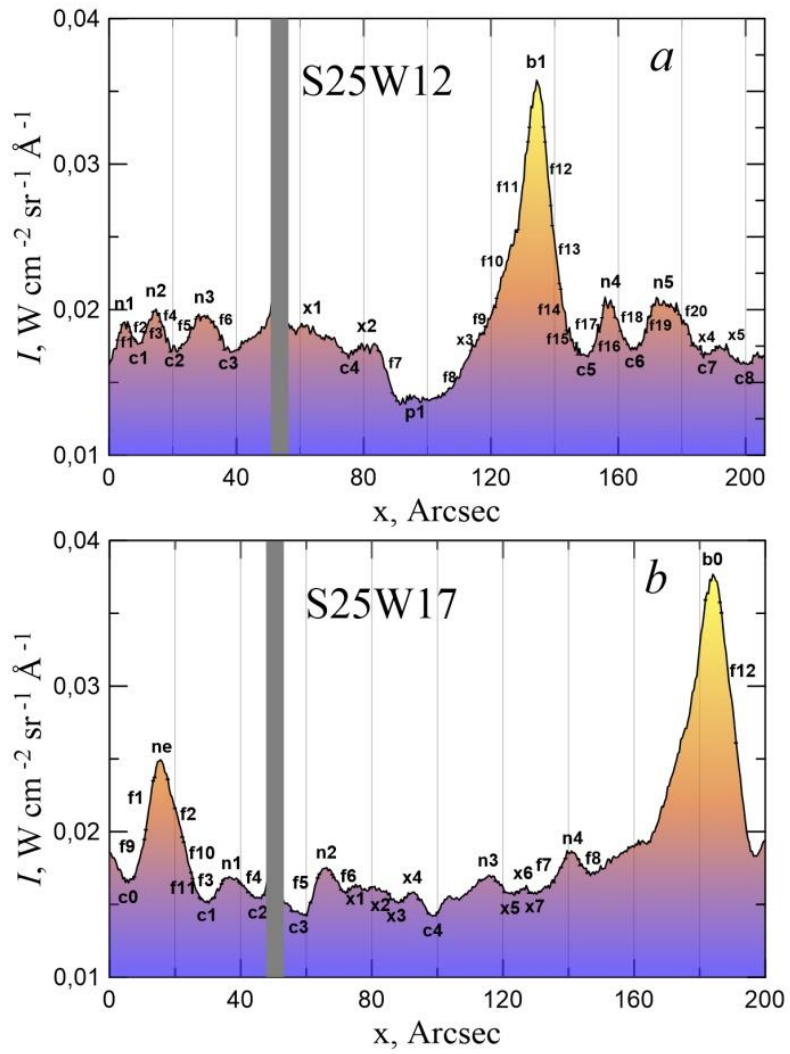

Figure 1. Time averaged intensity $I_{\mathrm{K} 3}$ along the spectrograph slit: S25W12 $(a)$; S25W17 (b). The selected chromospheric structures are denoted by letters and numbers. The gray vertical strip is a thread stretched across the spectrograph slit

«X » - structures with intermediate brightness, which do not belong to the above structures.

At each point of space, for the selected structures we constructed K Ca II line profiles. The line profile parameters of interest were calculated in an IDL code. All shifts in wavelength were calculated with respect to the nominal center of the K Ca II line. Wavelength calibration was performed using the FTS atlas [Brault, Neckel, 1987]. For several (10-12) reference lines identified in the spectrum and in the atlas, we calculated line centers and performed polynomial fitting of pixel numbers with wavelengths. The K Ca II line nominal center thus calculated was used as the zero point of the wavelength scale.

\subsection{Improvement of the determination tech- nique for $K_{1}$ and $K_{2}$}

The K Ca II line profiles observed in the quiet chromosphere not always have pronounced $\mathrm{K}_{2}$ peaks and $\mathrm{K}_{1}$ minima in contrast to profiles of the enhanced network and plages. Figure 2, $a$ illustrates a $\mathrm{K}$ line profile with pronounced $K_{2}$ peaks and $K_{1}$ minima. This profile pertains to the chromospheric structure with enhanced brightness. The $\mathrm{K}_{2}$ and $\mathrm{K}_{1}$ features for such profiles are found quite simply in IDL.

A different situation arises with the profiles such as in Figure 2, $b$.

This profile is associated with one of the dark structures of S25W17 (internetwork «c1»). There are no $\mathrm{K}_{2}$ peaks there. Instead of them there are small features with a less steep intensity variation, which resemble short plateaus
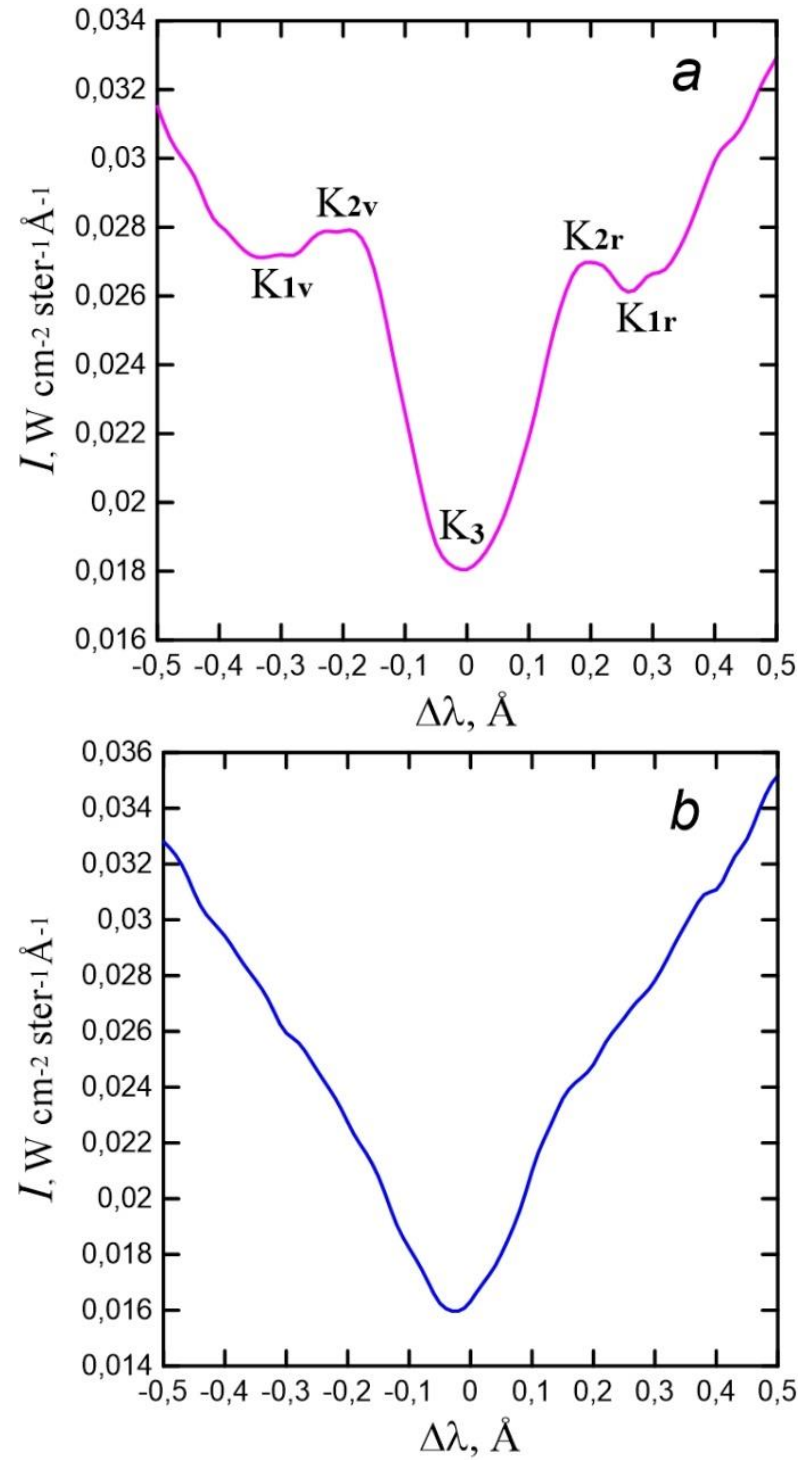

Figure 2. K Ca II line profiles in S25W17: for the chromospheric structure with enhanced brightness $(a)$; for the internetwork "c1" (b)

against a steep variation in the wing intensity. There are also profiles with almost monotonous intensity variation throughout the wing. We observed such reversal-free profiles in S25W12; they are described in Paper I. In such cases, we can speak of the location of the $K_{1}$ and $K_{2}$ features only under some assumptions. Nonetheless, we need information on these features to find the relationship between them and those profile features that are formed at other heights in the atmosphere.

In Paper I, we have already pointed out the difficulties related to computer processing of profiles with faint peaks. The IDL code used in Paper I could not always cope with calculations in $K_{1}$ and $K_{2}$. In those cases, we had to change the algorithm to fit it to each individual profile. This way is of little use for processing a large amount of observational data. To date, we have modified the algorithm, which allowed us to work with a uniform approach when processing the profiles with faint peaks.

Testing has shown that the most accurate result in determining the location of $\mathrm{K}_{1}$ and $\mathrm{K}_{2}$ can be obtained 
when using the second derivative of the $\mathrm{K}$ line intensity - $d^{2} I / d(\Delta \lambda)^{2}$. Grossmann-Doerth et al. [1974] employed the derivatives $d^{2} I / d(\Delta \lambda)^{2}$ for the same purpose, but the authors only briefly mentioned this in their paper, without describing the technique.

Using this approach involves two steps.

Step I. Search for extrema of the K Ca II intensity $I(\Delta \lambda)$ corresponding to $K_{1}$ and $K_{2}$ in a given wavelength range. The search range is set close to the mean value of $\Delta \lambda_{\mathrm{K} 2}$ we have obtained in Paper I, which is $\pm 0.21 \AA$ in the violet and red wings for both regions.

Step II. If under search conditions, the first step does not work, as the location of $\mathrm{K}_{2}$ and $\mathrm{K}_{1}$ we take values of $\pm 0.21 \AA$ and $\pm 0.30 \AA$ respectively. The value $\Delta \lambda_{\mathrm{K} 1}$ $\approx 0.30 \AA$ often characterizes two-peak profiles in a quiet region for which it is readily determined in IDL (e.g., our Figure 2, a). It is also known from the literature (see, e.g., [Liu, 1974, Table; Jensen, Orral, 1963, Figures 3, 7; Grossmann-Doerth et al., 1974]).

To implement Step I, we find the second derivative $d^{2} I / d(\Delta \lambda)^{2}$. We adopt the DERIV procedure from the IDL library, which performs numerical differentiation through three-point, Lagrangian interpolation. The line profile is set in the range $\Delta \lambda= \pm 0.5 \AA$ and includes 101 points with a step of $0.01 \AA$ (see Figure $2, a, b$ ).

For this problem it is convenient to use values of the derivative $d^{2} I / d(\Delta \lambda)^{2}$, although when describing a technique it is sometimes more convenient to use curvature $k$, which reproduces the behavior of $d^{2} I / d(\Delta \lambda)^{2}$ and numerically differs from it by less than $1 \%$. Our goal is to find the points at which $\left|d^{2} I / d(\Delta \lambda)^{2}\right|$ on a given range $\Delta \lambda$ reaches its maximum value provided that between these points $d^{2} I / d(\Delta \lambda)^{2}$ changes sign. In other words, we observe a transition from the concave intensity curve to the convex one if we consider each wing in the direction from $\Delta \lambda= \pm 0.5 \AA$ to the line center.

In the violet wing, the behavior of $d^{2} I / d(\Delta \lambda)^{2}$ is analyzed in the range $\Delta \lambda=(-0.27 \div-0.16) \AA$. In the red wing, the behavior of $d^{2} I / d(\Delta \lambda)^{2}$ is analyzed in the range $\Delta \lambda=(0.16 \div 0.30) \AA$. The maximum value of $d^{2} I / d(\Delta \lambda)^{2}$ in these ranges $\Delta \lambda$ indicates a point identified with the location of a $\mathrm{K}_{1}$ minimum. The minimum value of $d^{2} I / d(\Delta \lambda)^{2}$ indicates a point that corresponds to the location of a $\mathrm{K}_{2}$ peak.

Figure 3 presents the result of computations for the red wing of the profile illustrated in Figure 2, $b$. The blue line shows the intensity $I(\Delta \lambda)$ in the search range; the green line depicts the behavior of the second derivative.

The minimum value of $d^{2} I / d(\Delta \lambda)^{2}$ is at $\Delta \lambda=0.22 \AA$, which corresponds to the $\mathrm{K}_{2 \mathrm{r}}$ location. Maximum $d^{2} I / d(\Delta \lambda)^{2}$ is at $\Delta \lambda=0.25 \AA$ and indicates the $K_{1 \mathrm{r}}$ location.

Applying this method to profiles with faint peaks has shown that the method has a relatively high sensitivity to slight intensity variations in the line wing. For example, at the curvature $k \approx|0.2|$ a picture similar in quality to Figure 3 may emerge, but at such small $k$ this bending cannot be considered as a peak. For a weak peak we take

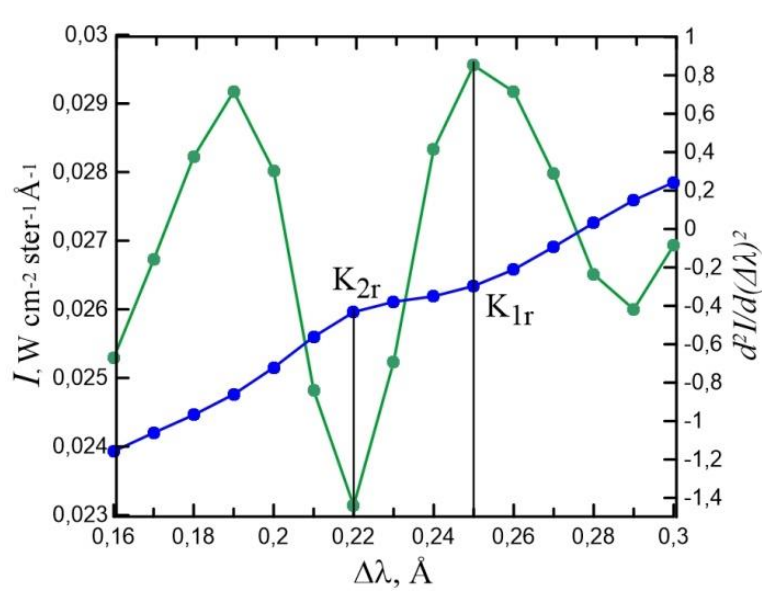

Figure 3. Result of determination of $\mathrm{K}_{1}$ and $\mathrm{K}_{2}$ positions for the red wing of the $\mathrm{K}$ line profile shown in Figure 2, $b$. Blue color is the intensity, green color is the second derivative of the intensity in the search range. Vertical lines show $\mathrm{K}_{1}$ and $\mathrm{K}_{2}$ locations

a wing part such that $k \geq|0.8|$. An example of this profile can be the profile in Figure 2, $b$. If in both wings of the profile the given condition is not valid, the profile is considered reversal-free.

Notice that profiles with well-defined peaks often have a larger distance between $\mathrm{K}_{2}$ and $\mathrm{K}_{1}$ features than those with faint peaks. We can see this, for example, when comparing the profiles depicted in Figure 2, $a, b$. So, in Figure 2, $a$ the difference between $\Delta \lambda_{\mathrm{K} 2}$ and $\Delta \lambda_{\mathrm{K} 1}$ is approximately $\approx \pm 0.08 \AA$, whereas in Figure $2, b$ it is $\approx \pm 0.03 \AA$.

\section{CORRELATIONS BETWEEN INDIVIDUAL PROFILE PARAMETERS}

We have explored possible relationships between the following parameters:

- $\quad I_{\mathrm{K} 1 \mathrm{v}}, I_{\mathrm{K} 1 \mathrm{r}}$ - intensities of the violet and red minima respectively;

- $\Delta \lambda_{\mathrm{K} 1 \mathrm{v}}$ and $\Delta \lambda_{\mathrm{K} 1 \mathrm{r}}-$ shifts of the $\mathrm{K}_{1 \mathrm{v}}$ and $\mathrm{K}_{1 \mathrm{r}}$ minima with respect to the nominal line center;

- $\quad I_{\mathrm{K} 2 \mathrm{v}}, I_{\mathrm{K} 2 \mathrm{r}}$ - intensities of the violet and red peaks;

- $\Delta \lambda_{\mathrm{K} 2 \mathrm{v}}$ and $\Delta \lambda_{\mathrm{K} 2 \mathrm{r}}$ - shifts of the $\mathrm{K}_{2 \mathrm{v}}$ and $\mathrm{K}_{2 \mathrm{r}}$ peaks with respect to the nominal line center;

- $\quad I_{\mathrm{K} 3}-$ a minimum intensity in the line center;

$\Delta \lambda_{\mathrm{K} 3}$ - a shift of the $\mathrm{K}_{3}$ minimum along the wavelength axis with respect to the nominal line center;

- $\quad S E P_{\mathrm{K} 1}$ - the separation of $\mathrm{K}_{1}$ minima equal to $\Delta \lambda_{\mathrm{K} 1 \mathrm{r}}-\Delta \lambda_{\mathrm{K} 1 \mathrm{v}}$

- $\quad S E P_{\mathrm{K} 2}$ - the separation of $\mathrm{K}_{2}$ peaks equal to $\Delta \lambda_{\mathrm{k} 2 \mathrm{r}}-\Delta \lambda_{\mathrm{K} 2 \mathrm{v}}$.

Mean values of the parameters are listed in Table. For all possible combinations of pairs of these parameters we constructed scatter plots, calculated mean correlation coefficients, and also built histograms showing how the parameter values are distributed for different chromospheric structures. Figures 4-6 give typical examples of scatter plots for some pairs of parameters. 
Mean parameter values. Shifts $\Delta \lambda$ and separations $S E P$ of peaks are given in $\mathrm{km} / \mathrm{s}$

\begin{tabular}{|c|c|c|c|c|c|c|c|c|c|c|c|c|}
\hline \multicolumn{13}{|c|}{ S25W12 } \\
\hline & $\Delta \lambda_{\mathrm{K} 1 \mathrm{r}}$ & $\Delta \lambda_{\mathrm{K} 1 \mathrm{v}}$ & $\Delta \lambda_{\mathrm{K} 2 \mathrm{r}}$ & $\Delta \lambda_{\mathrm{K} 2 \mathrm{v}}$ & $\Delta \lambda_{\mathrm{K} 3}$ & $I_{\mathrm{K} 1 \mathrm{r}}$ & $I_{\mathrm{K} 1 \mathrm{v}}$ & $I_{\mathrm{K} 2 \mathrm{r}}$ & $I_{\mathrm{K} 2 \mathrm{v}}$ & $I_{\mathrm{K} 3}$ & $S E P_{\mathrm{K} 1}$ & $S E P_{\mathrm{K} 2}$ \\
\hline «C» & 19.6 & -21.4 & 16.0 & -16.0 & 0.1 & 0.025 & 0.026 & 0.025 & 0.027 & 0.017 & 41.0 & 32.1 \\
\hline$\langle\mathrm{nn}\rangle$ & 20.8 & -23.5 & 15.8 & -16.1 & -0.3 & 27 & 0. & 0.028 & .029 & .020 & 44.3 & 31.9 \\
\hline$\langle b\rangle$ & 31.6 & -33.6 & 15. & -12.6 & 2.3 & 3 & 0.033 & 0.043 & 0.049 & 034 & 65.2 & 8.0 \\
\hline$\langle\langle f\rangle$ & 19.9 & -22.9 & 15.3 & -16.4 & -0.7 & 0.027 & 0.027 & 0.027 & 0.028 & .019 & 42.7 & 31.7 \\
\hline$\langle\mathrm{XX}\rangle$ & 18.8 & -21.2 & 15.1 & -16.1 & -0.6 & 0.025 & 0.026 & 0.025 & 0.026 & 0.018 & 40.0 & 31.5 \\
\hline «p» & 18.5 & -17.7 & 15.3 & -15.2 & -0.9 & 0.023 & 0.022 & 0.022 & 0.021 & 0.014 & 30.1 & 27.4 \\
\hline \multicolumn{13}{|c|}{ S25W17 } \\
\hline & $\Delta \lambda_{\mathrm{K} 1 \mathrm{r}}$ & $\Delta \lambda_{\mathrm{K} 11}$ & $\Delta \lambda_{\mathrm{K} 2 \mathrm{r}}$ & $\Delta \lambda_{\mathrm{K} 2 \mathrm{v}}$ & $\Delta \lambda_{\mathrm{K} 3}$ & $I_{\mathrm{K} 1 \mathrm{r}}$ & $I_{\mathrm{K} 1 \mathrm{v}}$ & $I_{\mathrm{K} 2 \mathrm{r}}$ & $I_{\mathrm{K} 2 \mathrm{v}}$ & $I_{\mathrm{K} 3}$ & $S E P_{\mathrm{K} 1}$ & $S E P_{\mathrm{K}}$ \\
\hline$\langle\mathrm{C}\rangle$ & 18.5 & -21.3 & 15.4 & -17.0 & -1.1 & 0.024 & 0.024 & 0.024 & 0.024 & 0.015 & 39.8 & 32.4 \\
\hline «n» & 19.3 & -21.8 & 15.3 & .2 & -0.2 & 0.025 & 0. & 0.025 & 0.027 & 0.018 & 41.1 & 30.5 \\
\hline$\langle\mathrm{kb}\rangle$ & 31.3 & -3 & 13.7 & 8 & 0.8 & 0.032 & 0.032 & 0.047 & 0.051 & 0.037 & 65.3 & 26.5 \\
\hline$\langle f\rangle$ & 19.5 & -22.2 & 15.3 & -16.2 & -0.6 & 0.025 & 0.026 & 0.026 & 0.027 & 0.018 & 41.7 & 31.4 \\
\hline$x \|$ & 18.2 & -20.0 & 15.5 & -15.8 & -0.5 & 0.024 & 0.024 & 0.024 & 0.025 & 0.016 & 38.2 & 31.4 \\
\hline
\end{tabular}
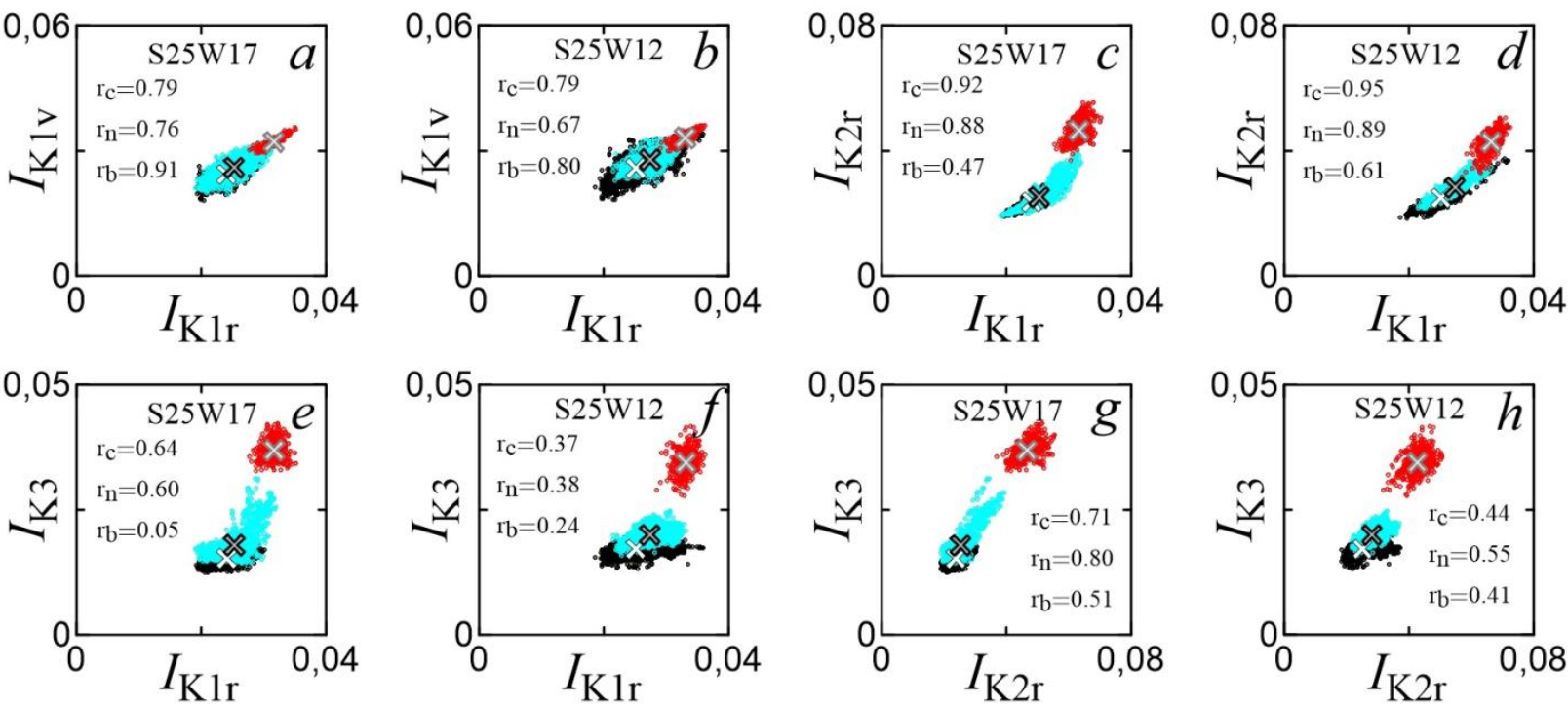

Figure 4. Scatter plots of intensities associated with various features of the K Ca II line profile formed at different heights of the solar atmosphere in S25W17 and S25W12. Color marks isolated chromospheric structures: black — for the «c» structure; blue - for «n»; red - for «b». Crosses indicate mean distribution values of «c» (white cross), «n» (dark-gray cross), «b» (lightgray cross). Intensities are given in the same units as in Figure 1
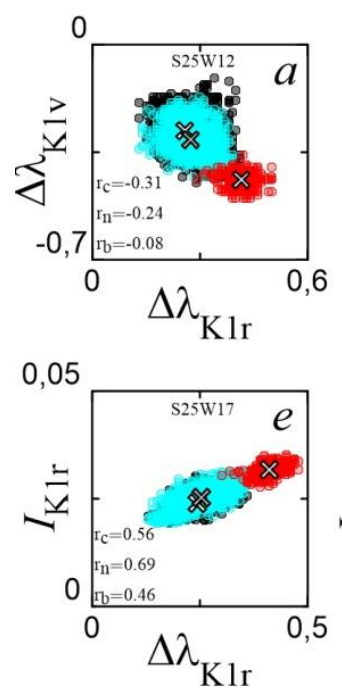
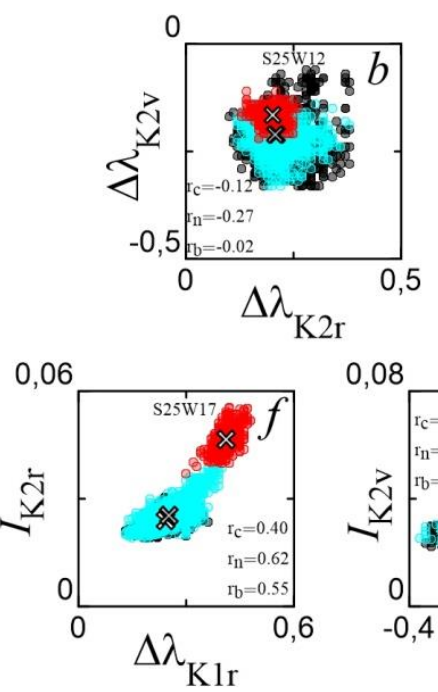

0,08

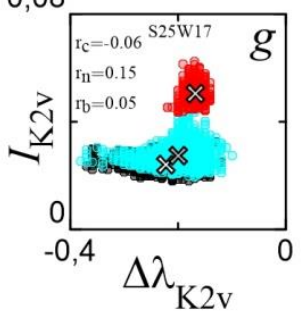

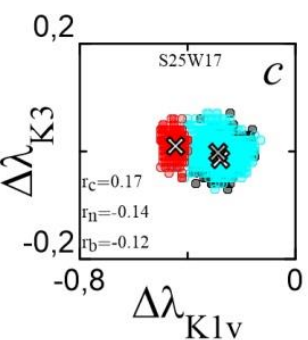

0,05

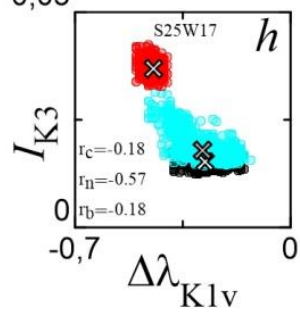

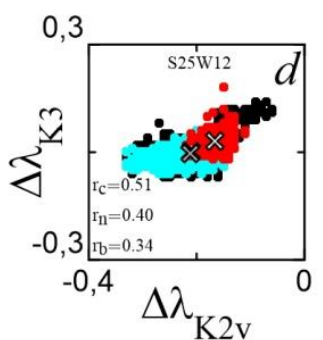

0,06

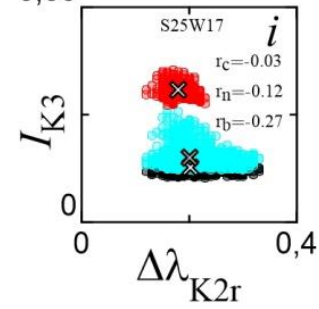

Figure 5. Scatter plots of shifts of different features of the K Ca II line profile. $\Delta \lambda$ is in angstroms. Designations are the same as in Figure 4 

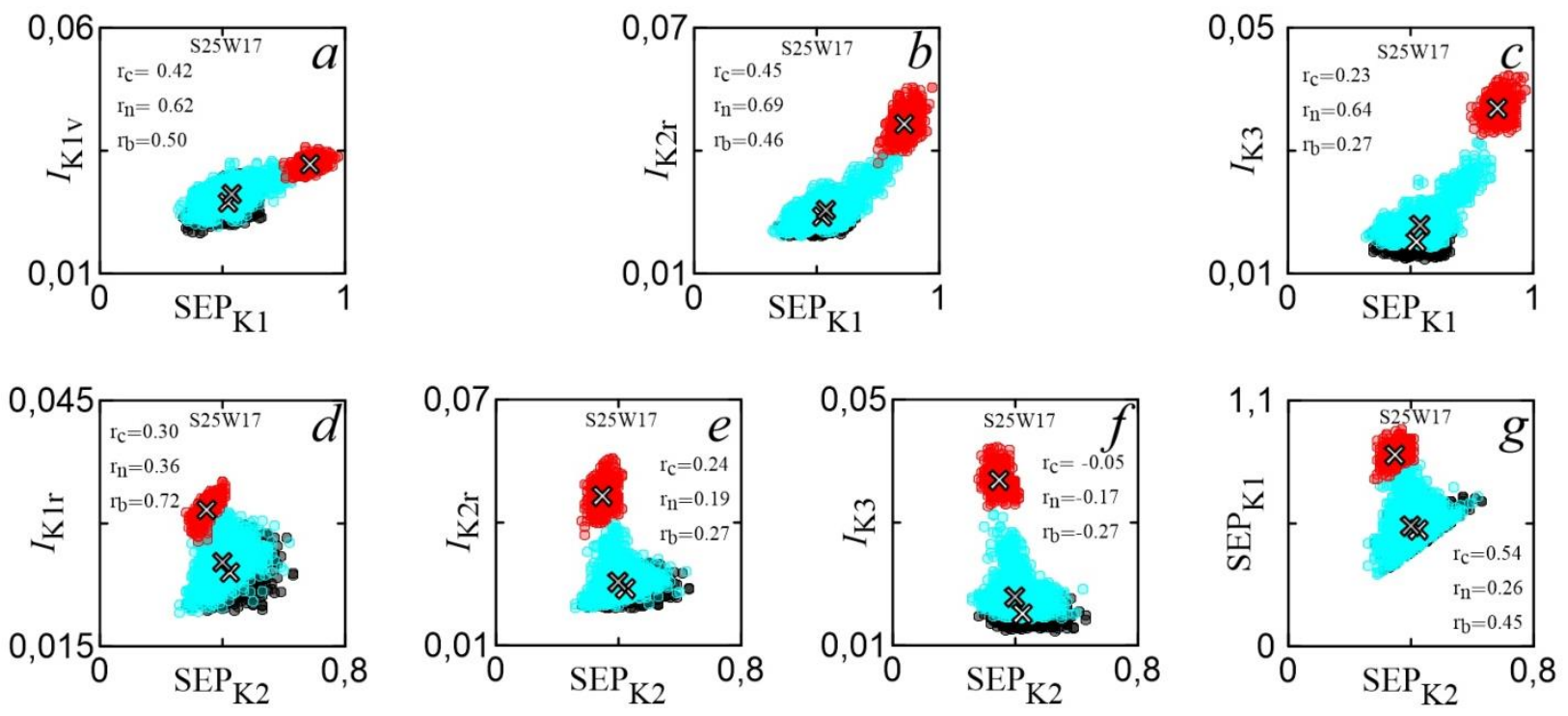

Figure 6. Scatter plots for separations of $\mathrm{K}_{1}$ minima and $\mathrm{K}_{2}$ peaks. Designations are the same as in Figure 4 . The separations are given in angstroms

Detailed examination of the results is easier to make by dividing the parameters into three groups: intensities, shifts of peaks and minima, separation of $\mathrm{K}_{1}$ minima and $\mathrm{K}_{2}$ peaks.

Intensities. We can note a high correlation between $I_{\mathrm{K} 1 \mathrm{v}}$ and $I_{\mathrm{K} 1 \mathrm{r}}$ (see Figure $4 a, b$ ), with the highest correlation in the plage and internetworks of the two regions. This correlation is expected because $K_{1}$ minima appear at close heights. Nevertheless, they are lower than the extremely high correlations obtained for the intensities in the corresponding wings at the $\mathrm{K}_{1}$ and $\mathrm{K}_{2}$ formation levels for the internetwork and network (Figure 4,c,d). The correlations between $I_{\mathrm{K} 1}$ and $I_{\mathrm{K} 2}$ are approximately equal for the violet and red wings (those for the violet wing are not shown). A lower correlation between $I_{\mathrm{K} 1}$ and $I_{\mathrm{K} 2}$ has been found for the plage from S25W17 ( 0.47 for the red wing and 0.38 for the violet one). Much lower correlations are observed for the intensities at the $\mathrm{K}_{1}$ and $\mathrm{K}_{3}$ levels (Figure 4, e,f). For the S25W17 plage the intensity correlations at the $\mathrm{K}_{1}$ and $\mathrm{K}_{3}$ levels are close to zero ( 0.05). Intensity correlations at the $\mathrm{K}_{1}$ and $\mathrm{K}_{3}$ levels for all selected structures are lower than those at the $\mathrm{K}_{2}$ and $\mathrm{K}_{3}$ levels (Figure 4, $g, h$ ).

Shifts of peaks and minima. We have found that the correlation between locations of $K_{1}$ minima in the violet and red wings is low (Figure 5,a), as well as the correlation between locations of $\mathrm{K}_{2}$ peaks in the violet and red wings (Figure 5, $b$ ). Both for $\mathrm{K}_{1}$ minima and for $\mathrm{K}_{2}$ peaks a higher correlation occurs in «c» and «n» structures. The correlation between locations of $\mathrm{K}_{1}$ minima and the location of the $\mathrm{K}_{3}$ minimum is also low (Figure 5 , c). The correlation between positions of $\mathrm{K}_{2}$ maxima and the $\mathrm{K}_{3}$ minimum for all the structures is higher than that for $\mathrm{K}_{1}$ minima (Figure 5,d).

Locations of $\mathrm{K}_{1}$ minima correlate with positions of corresponding $\mathrm{K}_{2}$ peaks $\left(\mathrm{K}_{1 \mathrm{v}}\right.$ and $\mathrm{K}_{2 \mathrm{v}}, \mathrm{K}_{1 \mathrm{r}}$ and $\mathrm{K}_{2 \mathrm{r}}$ ) (not shown). They are especially high in the red wing for «c» structures $\left(r_{c}>0.70\right.$ for S25W17), «X» structures with intermediate brightness $\left(\mathrm{r}_{\mathrm{x}} \approx 0.80\right.$ for $\left.\mathrm{S} 25 \mathrm{~W} 17\right)$, and, surprisingly, for the «p» structure with reduced brightness $\left(r_{p}>0.80\right)$. The last result cannot be considered reliable because of the reversal-free profiles of the «p» structure. Cross-correlations, for instance, between locations of the violet $\mathrm{K}_{1}$ minima and the red $\mathrm{K}_{2}$ peaks are very low $(\approx 0.02)$, increasing slightly in the plage (to 0.2 ).

High correlations for both regions were found between locations of $\mathrm{K}_{1}$ minima and $I_{\mathrm{K} 1}$ in respective wings (Figure $5, e$ ). There is a slightly lower but significant correlation between locations of $\mathrm{K}_{1}$ minima and $\mathrm{K}_{2}$ peak intensities (Figure 5, f). The cross-correlation, for example between $\Delta \lambda_{\mathrm{K} 1 \mathrm{v}}$ and $I_{\mathrm{K} 1 \mathrm{r}}$ is lower. The cross-correlation at the $\mathrm{K}_{2}$ formation level, for example, between $\Delta \lambda_{\mathrm{K} 2 \mathrm{r}}$ and $I_{\mathrm{K} 2 \mathrm{v}}$ is much lower than that at the $\mathrm{K}_{1}$ formation level (Figures are not given), as in the case of respective wings (Figure 5,e,g). Correlations between shifts of $\mathrm{K}_{1}$ minima and the intensity $I_{\mathrm{K} 3}$, shifts of $\mathrm{K}_{2}$ peaks and the intensity $I_{\mathrm{K} 3}$ are insignificant, with a few exceptions (see Figure 5, $h, i)$.

Separation of $K_{1}$ minima and $\mathrm{K}_{2}$ peaks. The scatter plots indicate high correlations between the separation of $K_{1}$ minima and the intensity at the same level of the atmosphere $I_{\mathrm{K} 1}$ for all the structures (Figure 6,a). There are also high correlations between the separation of $\mathrm{K}_{1}$ minima and $I_{\mathrm{K} 2}$ (Figure $6, b$ ). Correlations with $I_{\mathrm{K} 3}$ are lower (Figure $6, c$ ). The highest correlation between the separation of $\mathrm{K}_{2}$ peaks and $I_{\mathrm{K} 1}$ is for the plage «b» (Figure $6, d)$. The correlation of the separation of $\mathrm{K}_{2}$ peaks with $I_{\mathrm{K} 2}$ and $I_{\mathrm{K} 3}$ is low (Figure 6,e,f). High correlations between separations of peaks at the $K_{2}$ and $K_{1}$ formation levels occur for the structures «c» (see Figure 6,g) and «X» $\left(\mathrm{r}_{\mathrm{x}}=0.60\right.$, not shown $)$.

\section{RESULTS AND CONCLUSIONS}

The parameters found by the above technique have enabled the study of the solar atmosphere in a wide range of heights, from the photosphere to the middle chromosphere. The correlations we obtained allowed us to observe how close the relationship is between the calculated parameters at heights between the upper photosphere and the lower 
chromosphere $\left(\mathrm{K}_{1 \mathrm{v}}\right.$ and $\left.\mathrm{K}_{1 \mathrm{r}}\right)$ and the parameters in the lower chromosphere $\left(\mathrm{K}_{2 \mathrm{v}}\right.$ and $\left.\mathrm{K}_{2 \mathrm{r}}\right)$ and in the middle chromosphere $\left(\mathrm{K}_{3}\right)$.

The correlations between intensities at the $\mathrm{K}_{1}$ and $\mathrm{K}_{3}$ levels (Figure $4, e, f$ ) for all the structures selected appeared to be lower than those at the $\mathrm{K}_{2}$ and $\mathrm{K}_{3}$ levels (Figure $4, g, h$ ). By recognizing that the intensity $I_{\mathrm{K} 3}$ does not reproduce the local temperature of the middle chromosphere, we can assume that $I_{\mathrm{K} 3}$ should correlate with the intensity in lower layers of the solar atmosphere, where the source function is quite closely related to the Planck function. It would seem to correlate with $I_{\mathrm{K} 1}$. Our observations are inconsistent with this picture. Perhaps we need another method for comparing values at these levels, which takes into account the complex process of radiative transfer.

The distributions obtained for $I_{\mathrm{K} 1 \mathrm{v}}$ and $I_{\mathrm{K} 2 \mathrm{v}}$ (Figure 7, $a, b)$ are constructed for the $\mathrm{S} 25 \mathrm{~W} 17$ structures «c», «n», and «b». It is obvious that to structures with higher magnetic field strength correspond higher intensities at the $\mathrm{K}_{1}$ and $\mathrm{K}_{2}$ formation levels, i.e. in the upper photosphere and lower chromosphere. A similar result for $I_{\mathrm{K} 1 \mathrm{v}}$ and $I_{\mathrm{K} 2 \mathrm{v}}$ was obtained in the work [Leenaarts et al., 2018] from filter observations of structures with different brightness in an active region.

We have found a very weak correlation between shifts of $\mathrm{K}_{1}$ minima in the violet and red wings $-\Delta \lambda_{\mathrm{K} 1 \mathrm{v}}$ and $\Delta \lambda_{\mathrm{K} 1 \mathrm{r}}$ (Figure 5,a). The same situation is true for $\mathrm{K}_{2}$ peaks, i.e. for $\Delta \lambda_{\mathrm{K} 2 \mathrm{v}}$ and $\Delta \lambda_{\mathrm{K} 2 \mathrm{r}}$ (Figure 5,b). Perhaps this is due to
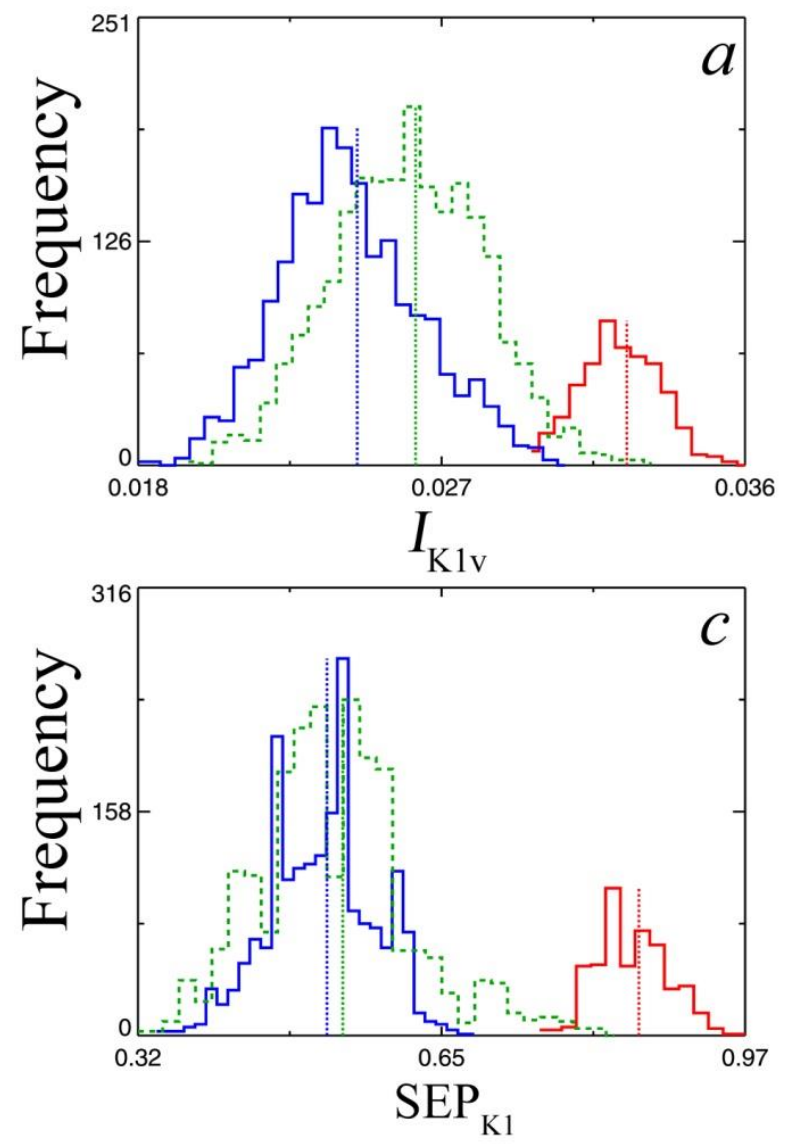

the fact that at levels of formation of $\mathrm{K}_{1}$ minima and $\mathrm{K}_{2}$ peaks random wave motions prevail rather than systematic. Moreover, the weak correlation may be due to the difference between heights of formation of the violet and red wings. Using results of the $3 \mathrm{D}$ simulation carried out by Bjørgen et al. [2018] and by the example of three models, the authors have shown that the $\mathrm{K}_{2 \mathrm{r}}$ peak appears slightly lower than the $\mathrm{K}_{2 \mathrm{v}}$ peak. Consequently, in the layers of $\mathrm{K}_{2 \mathrm{v}}$ and $\mathrm{K}_{2 \mathrm{r}}$ formation the dynamical processes occurring at a given moment of time may be different. This assumption is also confirmed by the higher correlation between locations of corresponding $\mathrm{K}_{1}$ minima and $\mathrm{K}_{2}$ peaks (e.g., $\Delta \lambda_{\mathrm{K} 1 \mathrm{v}}$ and $\Delta \lambda_{\mathrm{K} 2 \mathrm{v}}$ ) than the crosscorrelation between $\Delta \lambda_{\mathrm{K} 1 \mathrm{v}}$ and $\Delta \lambda_{\mathrm{K} 2 \mathrm{r}}$.

Results of our calculations for the separation of minima $S E P_{\mathrm{K} 1}$ have shown that an increase in the intensity in structures with a stronger magnetic field is accompanied by a broadening of the profile at the $\mathrm{K}_{1}$ formation level relative to the structures with a weaker magnetic field. Figure 7, $c$ shows distributions of $S E P_{\mathrm{K} 1}$ for the «c», «n», «b» structures of S25W17. Mean values of $\mathrm{SEP}_{\mathrm{K} 1}$ are seen to be greater for structures with a stronger magnetic field.

A similar separation of $K_{1}$ minima is noted in the work [Grossmann-Doerth et al., 1974], based on spectral observations of the $\mathrm{K} \mathrm{Ca}$ II line for regions in internetworks and on their boundaries (see Figure 7 and Table II therein).
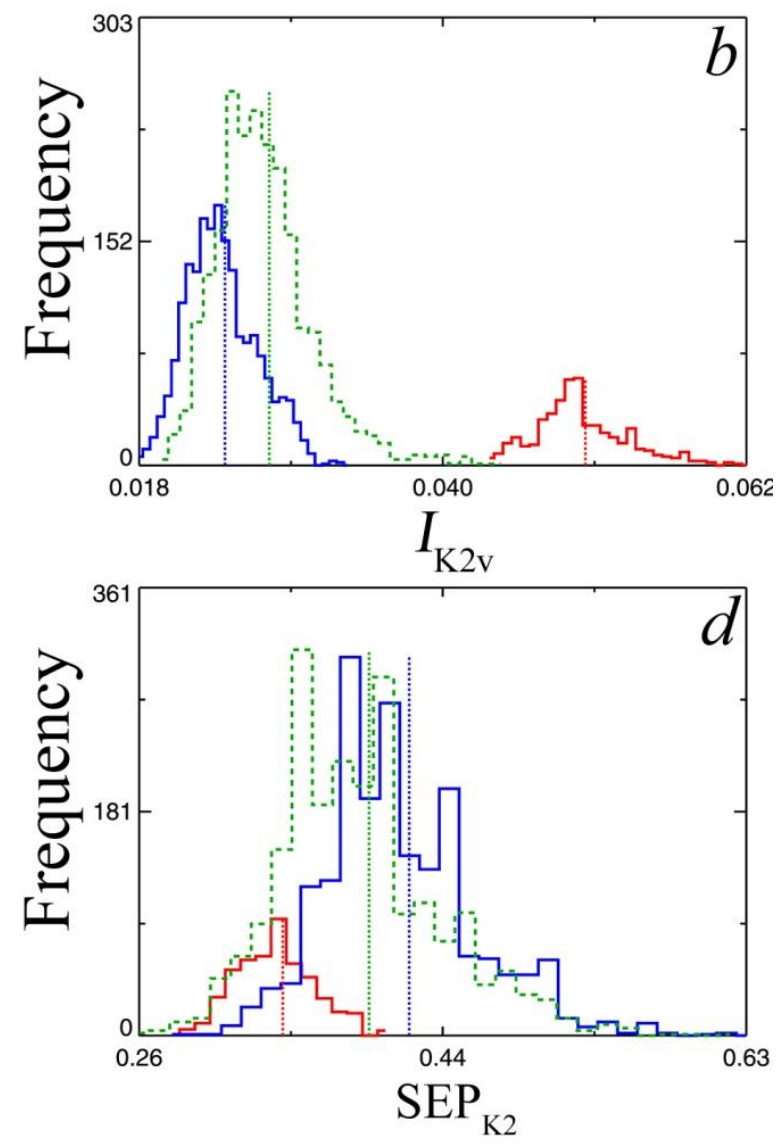

Figure 7. Histograms of $I_{\mathrm{K} 1 \mathrm{v}}, I_{\mathrm{K} 2 \mathrm{v}}$, separations of minima $S E P_{\mathrm{K} 1}$, separations of peaks $S E P_{\mathrm{K} 2}$ for $\mathrm{S} 25 \mathrm{~W} 17$. Distribution of the structures «c» is marked with blue; «n», with green; and «b», with red. Vertical lines indicate mean values of the distributions 
A qualitatively similar distribution for the separation of $\mathrm{K}_{1}$ minima have been obtained in [Leenaarts et al., 2018].

Comparison between our values of $S E P_{\mathrm{K} 1}$ for an internetwork and the separations of $\mathrm{K}_{1}$ minima derived from observations of a quiet region in [Bjørgen et al., 2018] shows that results of both studies are consistent with each other (see their Figure 12). The mean values of $S E P_{\mathrm{K} 1}$ listed in Table fall into the central part of the distribution for the separation of $\mathrm{K}_{1}$ minima in Figure 12 of those authors.

At the $\mathrm{K}_{2}$ formation level, the situation differs from that at the $\mathrm{K}_{1}$ formation level. There is an inverse relationship of $S E P_{\mathrm{K} 2}$ with $I_{\mathrm{K} 1}, I_{\mathrm{K} 2}$, and $I_{\mathrm{K} 3}$ (Figure $6, d-f$ ). Distributions of $S E P_{\mathrm{K} 2}$ for the $\langle(\mathrm{c}\rangle,\langle\langle\mathrm{n}\rangle,\langle\mathrm{b}\rangle$ structures are shown in the histogram (Figure 7,d). The mean values of $S E P_{\mathrm{K} 2}$ for the structures with a stronger magnetic field are seen to be smaller than those for the structures with a weak magnetic field. This is particularly noticeable for the plage. For it, not only the mean $S E P_{\mathrm{K} 2}$ but also the entire distribution is in the left wing of the lowest values of distributions for «c» and «n». A similar result has been obtained in the work [Kianfar et al., 2020], carried out on the basis of broadband images in the $\mathrm{H}$ and $\mathrm{K} \mathrm{Ca}$ II line cores. The authors have found that the separation of $K_{2}$ peaks in a bright structure is much smaller than outside of it. They have assumed that this may indicate either a lower microturbulent velocity in bright structures or the onset of chromospheric temperature rise located higher up.

Our $S E P_{\mathrm{K} 2}$ values are consistent with those obtained in [Bjørgen et al., 2018] for the separation of $K_{2}$ peaks from observations in a quiet region. Both mean $S E P_{\mathrm{K} 2}$ and most individual values are quite high, over $22 \mathrm{~km} / \mathrm{s}$. They fall into the central part of the distribution shown in Figure 12 [Bjørgen et al., 2018].

Summing up the results of our work, let us note the following.

The intensities that are observed in the lower and middle chromosphere are more closely linked to each other than the intensities in the upper photosphere and middle chromosphere.

Structures with a stronger magnetic field are brighter in the upper photosphere and lower chromosphere.

The weak relationship between $\Delta \lambda_{\mathrm{K} 1 \mathrm{v}}$ and $\Delta \lambda_{\mathrm{K} 1 \mathrm{r}}$ and between $\Delta \lambda_{\mathrm{K} 2 \mathrm{v}}$ and $\Delta \lambda_{\mathrm{K} 2 \mathrm{r}}$ may be determined by the significant role of random motions relative to systematic wave motions or by the difference between heights of formation of the violet and red wings, or by both factors.

In the profiles that belong to the structures with a stronger magnetic field, the separation of $\mathrm{K}_{1}$ minima is greater than that in the structures with a weaker magnetic field.

There is an inverse relationship between the separation of $\mathrm{K}_{2}$ peaks and $I_{\mathrm{K} 1}, I_{\mathrm{K} 2}, I_{\mathrm{K} 3}$, as compared to the separation of $\mathrm{K}_{1}$ minima. Mean values of $S E P_{\mathrm{K} 2}$ for the structures with a stronger magnetic field are smaller than those for the structures with a weaker field. It should be noted that in contrast to the statistics on «c» and «n», the statistics on «b» is small. Obviously, the correct statements about the profile behavior in a strong magnetic field of a plage require extra data for comparison. It is the aim of our further research.
We thank referees for their very helpful comments.

The work was performed with budgetary funding of Basic Research program II.16. The results were obtained using the equipment of Center for Common Use «Angara» [http://ckp-rf.ru/ckp/3056].

\section{REFERENCES}

Bjørgen J.P., Sukhorukov A.V., Leenaarts J., Carlsson M., de la Cruz Rodriguez J., Scharmer G.B., Hansteen V.H. Three-dimensional modeling of the $\mathrm{Ca}$ II $\mathrm{H}$ and $\mathrm{K}$ lines in the solar atmosphere. Astron. Astrophys. 2018, vol. 611, A62. DOI: 10.1051/0004-6361/201731926.

Brault, J.W., Neckel, H.: Spectral Atlas of Solar Absolute Disk-Averaged and Disk-Center Intensity from 3290 to 12510 A. 1987. URL: [http://www.hs.uni-hamburg.de/forsch/einbl/ html] (accessed June 5, 2020).

Grigoryeva S.A., Turova I.P., Ozhogina O.A. Studying Ca II $\mathrm{K}$ line profile shapes and dynamic processes in the solar chromosphere at the base of a coronal hole. Solar Phys. 2016, vol. 291, pp. 1977-2002. DOI: 10.1007/s11207-016-0951-9.

Grossmann-Doerth U., Kneer F., von Uexküll M. Properties of the solar Ca II K line at high spatial resolution. Solar Phys. 1974, vol. 37, pp. 85-97.

Jensen E., Orral F.Q. Observational study of macroscopic inhomogeneities in the solar atmosphere. IV. Velocity and intensity fluctuations observed in the $\mathrm{K}$ line. Astrophys. $J$. 1963, vol. 138, pp. 252-270.

Kianfar S., Leenaarts J., Danilovic S., de la Cruz Rodriguez J., Diaz Baso C.J. Physical properties of bright Ca II K fibrils in the solar chromosphere. Astron. Astrophys. 2020, vol. 637, A1. DOI: 10.1051/0004-6361/202037572.

Leenaarts J., de la Cruz Rodriguez J., Danilovic S., Scharmer G., Carlsson M. Chromospheric heating during flux emergence in the solar atmosphere. Astron. Astrophys. 2018, vol. 612, A28. DOI: 10.1051/0004-6361/201732027.

Liu S.-Y. Direct observational evidence for the propagation and dissipation of energy in the chromosphere. Astrophys. J. 1974, vol. 189, pp. 359-365.

Teplitskaya R.B., Ozhogina O.A., Turova I.P. Brightness distribution at the base of a coronal hole. Astron. Lett. 2006, vol. 32, no. 2, pp. 120-127. DOI: 10.1134/S106377370602006X.

Turova I.P., Grigoryeva S.A., Ozhogina O.A. Spatial and temporal variations of $\mathrm{K} \mathrm{Ca}$ II line profile shapes in different structures of the solar chromospheres. I. Features of individual profiles. Solar-Terr. Phys. 2018, vol. 4, iss. 4, pp. 3-11. DOI: 10.12737/stp-44201801.

URL: http://ckp-rf.ru/ckp/305(accessed June 5, 2020).

How to cite this article

Turova I.P., Grigoryeva S.A., Ozhogina O.A. Spatial and temporal variations of $\mathrm{K} \mathrm{Ca}$ II line profile shapes in different structures of the solar chromosphere. II. Determination technique and correlation relationships between the K Ca II line parameters for $\mathrm{K}_{1}$ and $\mathrm{K}_{2}$ features. SolarTerrestrial Physics. 2020. Vol. 6. Iss. 4. P. 10-16. DOI: 10.12737/stp64202002. 